individuals affected with epilepsy. We feel that there is an unmet need for ongoing education in this field which should be catered to the recipient's needs.

\section{AUDIT OF FIRST PAEDIATRIC ASSESSMENT OF CHILDREN REFERRED WITH SUSPECTED SEIZURES BEFORE AND DURING THE SARS-COV-2 PANDEMIC}

${ }^{1}$ Maymunah Khries, ${ }^{2}$ Helen Estyn-Jones. ' Great North Children's Hospital; ${ }^{2}$ Department of Paediatrics, Queen Elizabeth Hospital, Gateshead

10.1136/archdischild-2021-rcpch.5

Background Diagnosing epilepsy can be complex with misdiagnosis rates of $5-30 \%$. NICE guideline recommends all children and young people presenting with a suspected epileptic seizure to be seen by a specialist in the diagnosis and management of the epilepsies within 2 weeks of presentation. Timely specialist assessment aims to

- improve the detailed history upon which accurate diagnosis depends.

- facilitate appropriate subsequent management.

- support anxious parents.

Has Covid-19 pandemic added an extra challenge for specialist healthcare providers trying to achieve best practice?

Objectives

- To audit our specialist service for children and young people with suspected seizure(s) against NICE recommendation.

- To compare numbers of referrals and timing of first assessment during the pandemic with the same time period the year before.

- To look at the diagnostic outcomes following specialist assessment for first suspected seizure.

Methods

- Identification of 2 cohorts of children presenting to specialist outpatient service for suspected seizure between March-May 2019 and March-May 2020.

- Retrospective case notes analysis following first Paediatric assessment.

Results

- Number of referrals declined by more than $20 \%$ during the pandemic, especially from general practitioners.

- In 2019 cohort (pre-pandemic), 55\% of the cases were seen within 14 days of referral compared to $42 \%$ in 2020 cohort (during pandemic).

- More than half of the referrals were diagnosed as nonepileptic events after specialist review. However, there were less non-epileptic events diagnosed in 2020 compared to the previous year.

\section{Conclusions}

- The pandemic is likely to have reduced the number of referrals, possibly due to some families following the 'stay at home' message and not presenting at all, and GPs deciding against referral of vague or likely non-epileptic episodes.

- Delayed referral to first specialist assessment might have resulted from assumptions about service cancellations.

- Pandemic restrictions affect staffing and availability of spacious rooms for distanced consultations.

Discussion This audit examined the impact of first wave of the pandemic since when changes to our Paediatric service in general and the first seizure pathway may be helping meet the best practice challenge:

- Incorporating virtual platform in morning handover increases wider participation and opportunities to raise awareness of first seizure clinic.

- Remote access to trust systems means that referrals could be triaged quickly.

- Appointments are now mainly by 'attend anywhere' video or telephone, since event diagnosis is largely dependent on detailed history.

Further improvements are being considered:

- Work with IT to further develop electronic discharge documentation e.g. Drop-down menu of common presentations including 'first suspected seizure' with subsequent prompts such as 'eye-witness history', 'ECG' and 'first suspected seizure clinic referral'.

- Create email for epilepsy team to act as 'single point of access' to share triage, support communication and crosscover during leave.

- Continue to make investment in epilepsy service, including a Paediatric epilepsy nurse to support worried families, nonexpert colleagues and the service.

This poster could provide useful information to colleagues in primary and secondary care about our service and the clinical feedback may support training to improve confident diagnosis and management of common non-epileptic events such as syncope.

We aim to repeat this audit over the same time period in 2021.

\section{British Paediatric Allergy Immunity and Infection Group}

\section{A SERVICE EVALUATION OF PAEDIATRIC OUTPATIENT PARENTERAL ANTIBIOTIC THERAPY FOR THE MANAGEMENT OF DEEP BRAIN STIMULATION DEVICE INFECTIONS}

${ }^{1}$ Temitope Fisayo, ${ }^{2} J$ ennifer Handforth, ${ }^{3}$ Matthew Chico. ${ }^{1}$ London School of Hygiene and Tropical Medicine, King's College London; ${ }^{2}$ Evelina London Children's Hospital; ${ }^{3}$ London School of Hygiene and Tropical Medicine

\subsection{6/archdischild-2021-rcpch.6}

Background Dystonia is a movement disorder. Deep Brain Stimulation (DBS) is a surgical treatment for childhood dystonia wherein a DBS device is implanted in the brain. DBS devices may become infected. Paediatric Outpatient Parenteral Antibiotic Therapy (pOPAT) has been used to manage complex infections at home. This study evaluates the pOPAT service for the management of DBS device infections in a single centre. Objectives The goal of this project was to answer the following questions:

- Which pathogens infect the DBS devices in children who are subsequently managed by the pOPAT service?

- How are infections in children with DBS devices being managed under the pOPAT service?

- Does the pOPAT management prevent removal or revision of DBS devices following DBS device infection in children? 
- Does the pOPAT service improve antimicrobial stewardship in the management of DBS infections?

Therefore, the objectives of this study were:

- Identify the pathogens that infect the DBS devices of children managed by the pOPAT service

- Identify the antibiotics used to treat DBS device infections of children treated in the pOPAT service

- Measure the effect of the introduction of the pOPAT service on paediatric DBS device infection outcomes, including surgical revision and removal of DBS devices

- Measure the effect of the introduction of the pOPAT service on the antimicrobial stewardship involved in the management of DBS device infections in children.

Methods Paediatric Neurology and Paediatric Infectious Diseases service databases were searched for children with DBS devices implanted between 2000-2020. Descriptive statistics were used to characterise pOPAT. Logistic regression models were devised to estimate the odds ratios of DBS device removal and revision under pOPAT. Mann-Whitney $U$ and Fisher's exact tests were used to describe differences in antimicrobial stewardship.

Results Between 2000-2020, there were 211 children with DBS, 56 DBS device infections and 27 removals. Eleven of 56 infections were managed in pOPAT; 45/56 were managed in other settings. Adjusted odds ratio (aOR) for DBS device removal outside pOPAT was 9.11 (95\% CI 0.95, 86.9; $\mathrm{p}=0.055)$. DBS revision outside pOPAT: aOR was $5.43(95 \%$ CI $0.27,111 ; \mathrm{p}=0.272)$. The median length of antibiotic therapy was shorter in pOPAT (45 days vs 97 days; $\mathrm{p}=0.044)$. Managing infections in pOPAT was associated with better documentation of antibiotic therapy $(p=0.005)$ and swab sampling $(p=0.002)$. There was no difference $(\mathrm{p}=0.333)$ in the proportion of WHO AWaRe Access, Watch or Reserve antibiotics used to treat infections managed in pOPAT compared to other settings. Infections managed in pOPAT were more likely to be treated with ceftriaxone $(p=0.010)$ and teicoplanin $(p=0.008)$. There were ten different bacteria identified as the causative agent of DBS device infections in children. Of these, the most common were skin commensals, such as Staphylococcus aureus (21/38 results) and Staphylococcus epidermidis (4/38 results).

Conclusions The pOPAT service has conferred clear benefits in antimicrobial stewardship. There is suggestive evidence that pOPAT may be associated with DBS devices surviving infection without explantation. The service should continue to treat DBS device infections and maintain high standards in antimicrobial stewardship.

\section{Quality Improvement and Patient Safety}

\section{IMPACT OF IMPLEMENTATION OF REFERRAL PATHWAY FOR ABDOMINAL PAIN ON INITIAL ASSESSMENT TIME, INVESTIGATIONS REQUESTED, IMAGING PERFORMED AND EVENTUAL OUTCOME}

${ }^{1}$ Hina Rizvi, ${ }^{2}$ Chui Lai, ${ }^{2}$ Vijay Iyer, ${ }^{2}$ Anjum Gandhi. 'Birmingham Heartlands Hospital, Bordesley Green East; ${ }^{2}$ Birmingham Heartlands Hospital

10.1136/archdischild-2021-rcpch.7
Background In our trust, historically paediatric patients presenting with abdominal pain raising suspicion of appendicitis were referred directly to surgeons and assessed in the Paediatric Assessment Unit. These patients faced long wait times for management plan by surgeons:

- who also covered theatres/adult wards and intensive care

- waiting for blood and imaging investigation results before finalizing a plan.

This led to delays and affected flow of patients through the PAU and the ward capacity. The Paediatric team decided to bring about a change in the abdominal pain referral pathway by proposing to see all patients referred with abdominal pain and then if deemed appropriate to refer to surgeons.

We collected data over 2 periods; 2018 and 2020, with the implementation of the new pathway in 2019

Objectives To assess:

- time to first review by a team (paediatric vs surgical)

- percentage of patients

- with abdominal pain referred to each team

- who had blood tests and imaging requested by each team

- discharged, observed, referred and admitted by each team

Methods

- Sample period: 4 weeks in 2018 and 6 weeks in 2020

- Inclusion criteria: all paediatric patients referred with abdominal pain to PAU

- Exclusion criteria: anyone with previous appendectomy or re attending.

- Data collection: retrospective.

- Case notes: reviewed for referral record, review times, and initial diagnosis

- Electronic patient management system: reviewed for blood test and imaging investigation requested and discharge summaries

Results Primary care referral rose from $36 \%$ in 2018 to $47 \%$ in 2020 with a concurrent shift in ED referral from 53\% to $37 \%$ in 2020 showing the effect of the new pathway. Only $26 \%$ of referrals were made to Paediatric team in 2018 vs $87 \%$ in 2020

Majority of patients were seen within 4 hours by the Paediatric team in both episodes $86 \%$ vs $85 \%$. There was very slight improvement in Surgical team review time $78 \%$ vs $80 \%$.

There was an increase in discharges to $58 \%$ by the surgical team in 2020 from $35 \%$ in 2018. However, we also saw the increase in referral by the Paediatric team to surgical team from $7 \%$ to $33 \%$

There was an impressive reduction in blood investigation requested by the Surgical team from $90 \%$ to $58 \%$. Requests for imaging by surgical teams reduced from $33 \%$ to $17 \%$.

$88 \%$ of the referred patients with abdominal pain had medical diagnosis at discharge and did not need any surgical intervention

Conclusions Summary:

Re-auditing after implementing new Surgical abdominal pathway shows:

- Overall patient flow through PAU has improved as more patients are being discharged. 